\title{
Electroporation in Microfluidic Devices
}

\author{
Séverine Le Gac and Iris van Uitert
}

\begin{abstract}
Crossing the plasma cellular membrane for loading of exogenous substances or accessing the intracellular medium is essential for cell engineering and transfection, cell analysis, or controlled extraction of the cellular content. Various chemical and physical techniques have been developed to open up the cell membrane and allow molecular exchange between the extra- and intracellular environments. Electroporation, which relies on the use of a high external electric field to permeabilize the cell membrane, is the most popular physical technique: not only it avoids the use of viral material, but the cell transfection yield is also enhanced compared to chemical approaches. However, while electroporation is currently used on a daily basis for the transformation of a great variety of cells, it still suffers from a low success rate when it is performed in bulk in a cuvette, at the level of an mL-sized cell population. Furthermore, the use of high voltages in the $\mathrm{kV}$ range as required in such cuvettes gives rise to various issues, such as Joule heating, creation of bubbles through electrolysis of water, and generation of reactive species, which all compromise the success of the electroporation treatment. Using miniaturized and/or microfluidic devices helps solving these issues while enhancing the overall electroporation success rate, by bringing enhanced control on the process and requiring voltages as low as a few volts. In this chapter, after a short introduction to microfluidics, the unique features this technology can offer for cellular electroporation are discussed. Next, different classes of
\end{abstract}

\footnotetext{
S. Le Gac $(\bowtie)$

Applied Microfluidics for BioEngineering Research, MESA+ Institute for Nanotechnnology, MIRA Institute for Biomedical Engineering and Technical Medicine, University of Twente, Enschede, The Netherlands e-mail: s.legac@utwente.nl

I. van Uitert

Demcon bv, Enschede, The Netherlands

e-mail: iris.van.uitert@demcon.nl
} 
microfluidic devices for cell electroporation are presented, which are suitable for the treatment of individual cells or small cell populations. Finally, promising applications of microscale cellular electroporation are discussed.

\section{Keywords}

Microfluidics • Miniaturization • Single-cell electroporation • Flow-through devices $\bullet$ Integrated electrodes

\section{Contents}

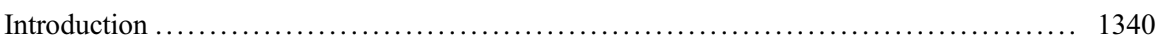

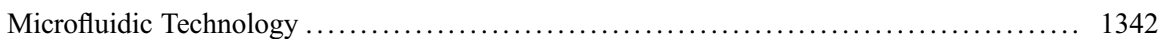

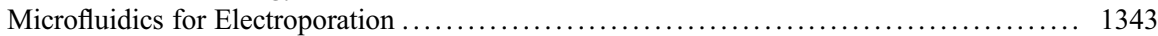

Classification of Microfluidic Devices for Electroporation ......................... 1345

Single-Cell Electroporation Devices ...................................... 1345

Flow-Through Electroporation Devices ................................... 1348

Promising Applications of Microfluidics for Cell Electroporation .................... 1352

Fundamental Research .................................................. 1353

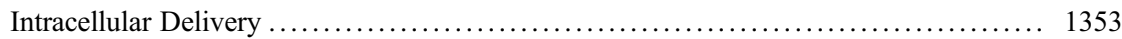

Food and Biotechnology Applications .................................. 1354

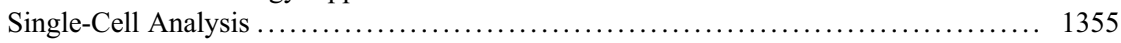

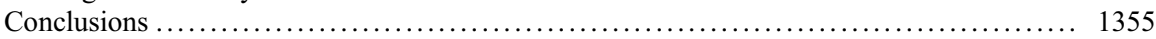

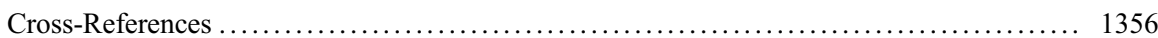

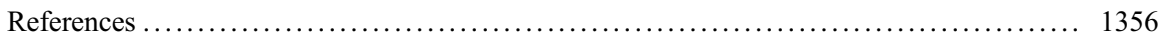

\section{Introduction}

Electroporation is a widely employed physical technique to transiently permeabilize cell membranes, and it finds multiple applications, e.g., for the introduction of foreign material into cells, for partial or entire extraction of the intracellular content, or for inactivation of microorganisms. Upon exposure to an external electric field, the cell transmembrane potential increases. Provided a threshold value ( $\triangleright$ Chap. 2, "Critical Electric Field and Transmembrane Voltage for Lipid Pore Formation in Experiments") of $0.2-1 \mathrm{~V}$ is reached, this increase is accompanied by the formation of conducting pores within a few microseconds, after rearrangement of the molecular structure of the membrane (Fig. 1). Depending on the strength of the electrical treatment, this process is reversible and aqueous pores reseal. Molecular transport can take place through these pores, this being governed mainly by diffusion for small species or involving more complex transportation mechanisms ( $\triangleright$ Chap. 23, "Gene Delivery by Electroporation In Vitro: Mechanisms") for plasmids or large entities.

Conventionally, electroporation is performed in bulk at the level of a cell population. In bulk electroporation, a cell suspension $\left(>10^{6}\right.$ cells, up to a few $\left.\mathrm{mL}\right)$ is introduced in a cuvette equipped with two facing electrodes, on which a high voltage is applied in the form of short pulses (exponentially decaying or square pulses, in the 


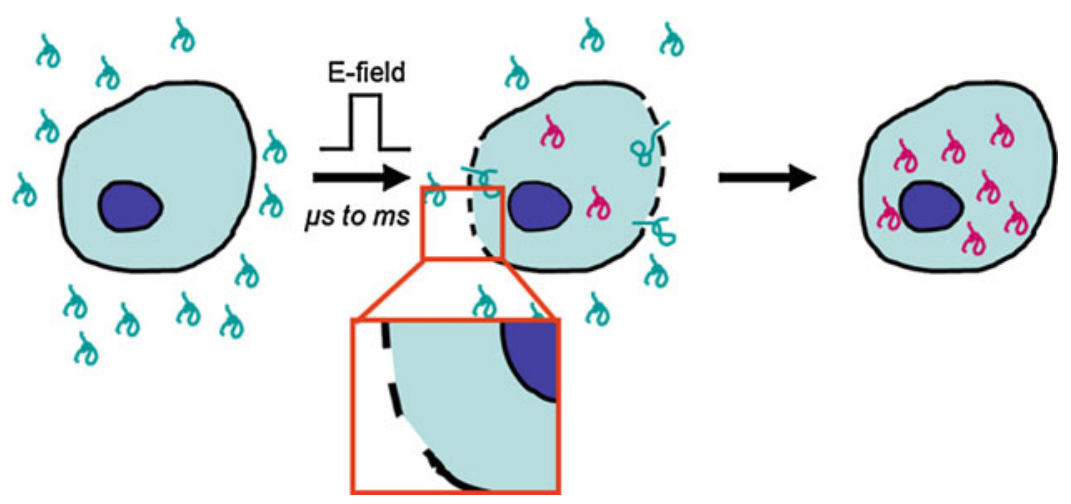

Fig. 1 Principle of cell electroporation. When a cell is exposed to short pulses of a high electric field, the cell transmembrane potential increases. If the latter reaches a critical value of $0.2-1 \mathrm{~V}$, pores are created in the cell membrane. These pores allow molecular exchange between the extraand the intracellular media and, for instance, loading into the cell of foreign substances for which the cell membrane is normally impermeable. In case of reversible electroporation, the pores in the cell membrane reseal (Single Cell Analysis, Single Cell Electroporation using Microfluidic Devices, 2012, page 66, S. Le Gac and A. van den Berg, "with permission of Springer")

low ms range). In such a cuvette, voltages in the $\mathrm{kV}$ range are applied to reach the required threshold for poration. Furthermore, in bulk electroporation, the viable poration rate, corresponding to cells being porated but remaining viable after the treatment, is low (40-70\%) (Chen et al. 2008). In general, three scenarios concomitantly take place, which explains this overall low success rate. In a first scenario, there is no membrane poration. In a second scenario, too many pores or too large pores are created so that the cell is not able to recover, which is known as irreversible poration. Finally, in the most favorable case, the membrane is permeabilized and reseals.

Bulk electroporation suffers from a number of issues, which explains this heterogeneity in the response of the cells to the electric field and the overall low success rate. First, the electric field is not homogeneous in the cuvette, and cells are randomly oriented with respect to the electric field direction, so that they experience different treatments. Next, the use of high voltages brings about a number of problems. Upon application of high voltages, reactive chemicals, which are toxic to the cells, are produced. Furthermore, Joule heating can induce a significant change of temperature in the cell suspension. Similarly, water electrolysis can happen, with the creation of bubbles, which can also result in a loss of cell viability. Finally, toxic metal ions released from the large plate electrodes also endanger cell viability, depending on the nature of the electrode material. Moreover, this cuvette-based approach is not optimal for the treatment of all kinds of cells. Adherent cells must undergo a (bio) chemical treatment to be placed in solution, which may damage the cell membrane and sensitize the cells to the electric field. Furthermore, rare cells, such as stem cells or induced pluripotent stem cells (iPSCs), are available in too limited amounts to be 
handled in an mL-sized cuvette. A promising approach to solve some of the aforementioned issues while bringing better control on the treatment to eventually enhance its success rate is to use microfluidic technology and miniaturized devices.

In this chapter, microfluidics is shortly introduced, and the unique features this technology has to offer for cell electroporation in comparison with a bulk process are discussed. Thereafter, different microfluidic-based strategies for cell electroporation are presented as two distinct categories of devices, which are especially suitable for single-cell electroporation and for flow-through treatments, respectively, and the specific advantages and limitations of these two types of devices are discussed. Finally, different fields of applications of cellular electroporation that can especially benefit from the use of microfluidic technology are briefly reviewed.

\section{Microfluidic Technology}

Microfluidics, as pointed out by Georges Whitesides, can be defined as "the science and technology of systems that process or manipulate small $\left(10^{-9}\right.$ to $\left.10^{-18} 1\right)$ amounts of fluids using channels with dimensions of tens to hundreds of micrometers" (Whitesides 2006). Microfluidic devices - also known as lab-on-a-chip devices - are miniaturized devices, which have a footprint of a few square centimeters. These devices include structures with dimensions in the 1-100 $\mu \mathrm{m}$ range such as channels or chambers, in which such small volumes of liquids can accurately be transported and processed. The field of microfluidics initially focused on the miniaturization of analytical devices. The motivations to use miniaturized devices for biomolecule and medical analysis were manifold, e.g., the possibility to work with small-sized samples (in the sub-microliter range), the rapidity and sensitivity of the analysis, and the portability of the systems to eventually yield autonomous point-of-care devices. Since their introduction in the 1990s, microfluidic devices have become more and more popular, and their applications have greatly diversified toward, for instance, the field of chemistry and synthesis, and also, more and more, for cell biology-related and biological research.

Since the field of microfluidics directly originated from those of microelectronics and microelectromechanical systems (MEMS), microfluidic devices have first been fabricated from silicon and silicon-based materials using processes from the microelectronics industry (i.e., photolithography, wet and dry etching, etc.) in a clean room environment. Glass quickly came in the picture and was preferred to silicon for its transparency and electrical insulating properties. As the field has been further evolving and expanding, materials and associated fabrication processes have been changing. Traditional materials now have to compete with polymers, which are much cheaper and already ubiquitous in laboratories. Polymer processing relies on alternative approaches such as replication or ablation techniques (Becker and Gartner 2008), which do not require having access to dedicate and expensive clean room facilities, but which can readily be implemented in a standard wet lab. 
The popularity of microfluidic devices is explained not only by the numerous advantages they present compared to their lab-scale counterparts but also by the fact that they offer new experimentation opportunities, especially in the fields of chemistry and cell biology. Among commonly cited advantages linked to their small dimensions are reduced consumptions of samples and reagents and associated lower operational costs. Similarly, all processes that scale with the dimensions of the device become more efficient and faster like mass transport, heat transfer, gas exchange, molecular diffusion, etc. For experimentation involving cells, the micrometer-sized structures make these devices ideal tools for the manipulation and isolation of individual cells and to conduct experimentation at the single-cell level (Le Gac and van den Berg 2010). Next to this, microdevices enable to emulate the confined environment cells experience in vivo. At the micrometer scale, flows also behave in a very different way than at the macrometer scale, which can be evaluated by examining the dimensionless Reynolds number $(R e)$. The Reynolds number compares inertial and viscous forces and indicates the transition from a turbulent to a laminar regime. In miniaturized devices, as a result of the increased surface-to-volume ratio, surface forces dominate bulk phenomena, and the flow is mostly in the laminar regime. Consequently, flows are predictable and highly controlled in microfluidic devices. A downside of a laminar flow regime however is that mixing only proceeds via molecular diffusion and is therefore not as efficient as when turbulences are involved. Altogether, microfluidic devices offer the possibility to accurately control any physical and chemical parameter both spatially and temporally. This ability is essential in biology to fine-tune the cellular microenvironment, as well as in chemistry to yield more homogeneous reaction mixtures. Finally, microfluidic devices present a high level of integration. One device can, for instance, comprise a series of identical systems (horizontal integration) for assay parallelization. Alternatively, a series of distinct operations can be implemented in one single device (vertical integration) for the accomplishment of a complete (analytical) process. Furthermore, microfluidic structures can be combined with add-on capabilities such as electrodes or sensors (smart integration), e.g., for electrical detection of cells or biomarkers, for controlling the temperature, or for fluid actuation.

\section{Microfluidics for Electroporation}

For cellular electroporation, microfluidic technology brings a number of attractive features. First, distances in microfluidic devices are in the micrometer range, and since the required electroporation voltage scales with the device dimensions, a few volts are typically sufficient to reach the $\mathrm{kV} / \mathrm{cm}$ electroporation threshold. Furthermore, microstructures can be designed to locally enhance and/or shape the electric field so that even lower voltages are required. For instance, channels can include constrictions in which the electric field is enhanced, since the E-field strength scales with the cross section of the channels. Alternatively, micrometer-sized structures can 
be added to isolate cells, which allows locally creating hot spots in electric field across the trapped cell. Similarly, electrodes can be directly integrated in the device and their shape and geometry optimized toward the same goal. For instance, saw-tooth electrodes in a microchannel have been reported to locally enhance the field strength (Lu et al. 2005). Furthermore, 3D electrode structures provide a more homogeneous electric field distribution across a channel compared with planar electrodes. Altogether, using microfluidics, the electroporation treatment becomes much milder, and all adverse effects originating from the use of high voltage, as normally encountered in bulk electroporation, are drastically reduced or entirely alleviated using such miniaturized devices.

As mentioned earlier, microfluidics lends itself well to single-cell experimentation, so that the electroporation treatment can be downscaled to the level of a single cell - or even to the subcellular level - by isolating individual cells at specific positions between two electrodes. This configuration brings about a high control on the electroporation treatment, and all parameters (electric field strength, duration of the treatment, etc.) can be adjusted for every single cell. Arguably, this single-cell approach opens the doors for a $100 \%$ theoretical success rate of the treatment. Furthermore, single-cell electroporation is particularly attractive for rare cells, which can be treated individually in a highly controlled and customized fashion, with a high success rate and no loss in viability. Finally, this single-cell approach is interesting to control molecular delivery and/or extraction following cell membrane poration, as discussed in more detail later in this chapter.

The high level of integration offered by microfluidics allows combining the electroporation treatment with another process. Cells can be tracked in situ to monitor the process of pore formation in real time and the outcome of the treatment, on a longer time scale. To that end, fluorescent probes are typically employed to instantaneously follow molecular transport across the membrane resulting from its successful permeabilization. Alternatively, the process of pore formation can be detected electrically, by monitoring the membrane conductance or the impedance ( $\triangleright$ Chap. 38, "Assessment of Electroporation by Electrical Impedance Methods") of individual cells, using either the same electrodes as for the electroporation or a second pair of electrodes. For longer-term examination, cells can be cultured in the same device and their state evaluated in situ after one or more days using bright-field and/or fluorescence microscopy. A common approach to validate a cell electroporation protocol consists of transfecting a plasmid coding for the green fluorescent protein (GFP); this strategy however implies keeping the cells in culture for about 1 day to verify not only that they have survived the treatment but also that they are functioning properly and able to produce proteins from the exogenous and transfected plasmids. This whole sequence can be implemented in a microfluidic device, with cells being tracked to examine the success of the electroporation/transfection treatment at the single-cell level. The electroporation treatment can finally be coupled to a cell-sorting step, e.g., using dielectrophoresis, either prior to electroporation to select cells to be exposed to the electric field or after, to isolate successfully porated cells. 


\section{Classification of Microfluidic Devices for Electroporation}

In this section, different microfluidic-based cell electroporation strategies are discussed to illustrate the potential of this technology for cell electroporation. Microfluidic devices for cell electroporation are classified in two distinct categories depending on how the treatment is performed: either at the single-cell level or using a flow-through configuration on cell populations. For each of the two approaches, the concept underlying the strategy is explained, and possible variations around the strategies are presented, together with a short discussion on the specific advantages of these respective devices and their possible limitations.

\section{Single-Cell Electroporation Devices}

\section{Principle}

Single-cell electroporation devices all rely on the isolation of individual cells, which are typically secured in trapping microstructures. These mechanical trapping sites consist of either microholes, which are located underneath the cells (Huang and Rubinsky 2001, 2003), or lateral trapping slits connected to another microchannel (Khine et al. 2005; Valero et al. 2008; van den Brink et al. 2011). In both cases, the trapping structures present a characteristic dimension of 2-3 $2 \mathrm{~m}$, which is several times smaller than the cell diameter. Importantly, these low micrometer-sized structures also allow shaping the electric field and creating hot spots of electric field across the isolated cells. Moreover, a microsystem can easily include arrays of these structures for the electroporation of a series of individual cells.

Trapping of the cells is a key step in this single-cell electroporation protocol. Typically, a negative pressure is applied across the trap after injection of the cell suspension in the device to attract and immobilize individual cells in the trapping sites. A too high negative pressure may damage the cells and increase their vulnerability and associated risks for cell death. On the contrary, if this force is too low, the cell may not be tightly immobilized in the trapping site and can easily escape. Furthermore, if a cell is loosely trapped, a leakage pathway is created so that a higher potential must be applied for cell poration. This leakage pathway also obviously prevents the electrical detection of pore formation.

Once the trap(s) is (are) filled, the cell(s) can be electroporated. To apply the electroporation voltage, electrodes are either integrated in the device or inserted in the access reservoirs. In the former case, electrodes can be placed in the close vicinity of each trapping site, so that a voltage of a few volts is typically needed to achieve cell electroporation. Furthermore, in this configuration, the electric field parameters can be adjusted for each individual cell, would the device include an array of trapping sites and individually addressed electrodes for each trapping site. Therefore, this strategy is particularly attractive to optimize the treatment for every single cell to eventually give a $100 \%$ (viable) poration yield. 


\section{Bottom Trapping of Cells}

A first type of single-cell electroporation devices includes a horizontal substrate with an array of micrometer-sized apertures in which cells are trapped. Two channels or chambers are placed above and underneath this perforated substrate to, respectively, introduce the cell suspension and apply a negative pressure for cell trapping.

The first microfluidic cell electroporation device worked along this principle of single-cell trapping in a horizontal microhole (Huang and Rubinsky 2001, 1999). This device, reported by Huang and Rubinsky, was composed of two silicon chambers separated by a horizontal $1-\mu \mathrm{m}$-thick silicon nitride membrane containing a $2-10-\mu \mathrm{m}$ diameter orifice (Fig. 2a). The electrodes, which were integrated in the device, consisted of $\mathrm{n}+$-polysilicon membranes placed in the two silicon chambers, and they were separated by $900 \mu \mathrm{m}$. The semiconductive and translucent properties of this material allowed both electrical and optical monitoring of the trapping and poration processes. Trapping of a cell and sealing of the cell in the orifice resulted in a great reduction of the current through the trap - so-called leakage current - while cell electroporation was accompanied by an increase in the conductance across the orifice. Using this device, the electroporation voltage was successfully lowered to a few $10 \mathrm{~s} \mathrm{~V}$ (single square pulse of $60 \mathrm{~ms}$ ).

Adaptations have been brought to this first device, which proved the concept of single-cell microfluidic electroporation while being limited to one single cell. Addition of another pair of electrodes allowed detecting cells arriving on the micro-orifice to trap them. After electroporation, reversing the pressure released the cell, so that another cell could be attracted in the orifice and subsequently treated (Huang and Rubinsky 2003). This planar configuration of orifices is easily amenable to parallelization to increase the poration throughput through the use of $2 \mathrm{D}$ arrays of orifices, in which populations of cells are immobilized and submitted to an electric field. However, in most of these planar devices, common electrodes are used for all orifices in the array so that the electric field treatment cannot be customized per cell, and similarly, the poration process cannot be monitored electrically.

\section{Lateral Trapping of Cells}

In a second configuration, trapping is achieved using lateral structures separating the channel, in which the cell suspension is injected, and another channel (Valero et al. 2008) or a series of individual channels (Khine et al. 2005; van den Brink et al. 2011), from which the negative pressure is applied (Fig. 2b, c). This lateral design lends itself better to electrical decoupling of the traps and to multiplexed single-cell electroporation. Furthermore, provided the different trapping sites are connected to individual channels, the lateral approach is also compatible with parallel single-cell analysis (van den Brink et al. 2011). A second main advantage of this lateral approach, when individual channels and electrodes are used, is that the electroporation process can be monitored electrically; thereby, as soon as pore formation is detected through a change in the measured membrane conductance, the electroporation voltage can be stopped. Similarly, individual channels and individually addressed electrodes can be employed to assist and enhance the introduction of 


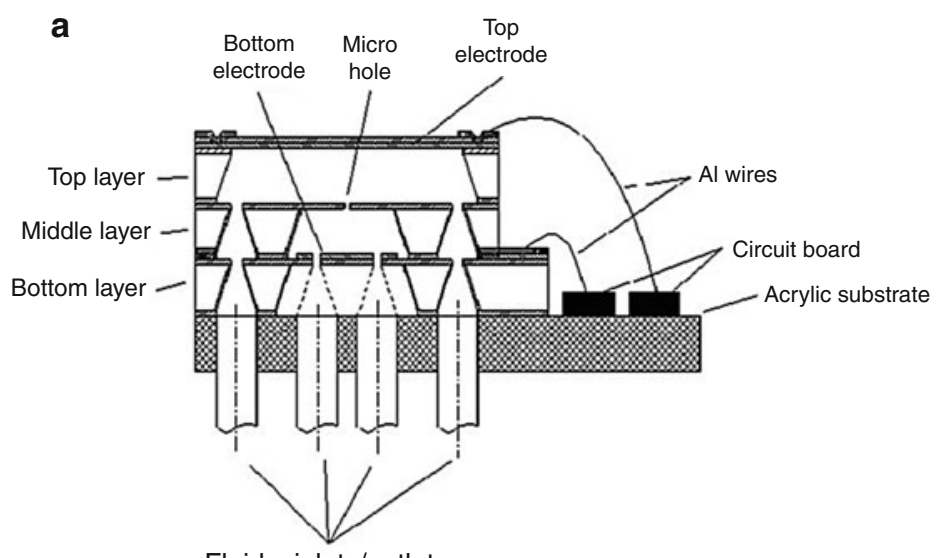

Fluids inlets/outlets
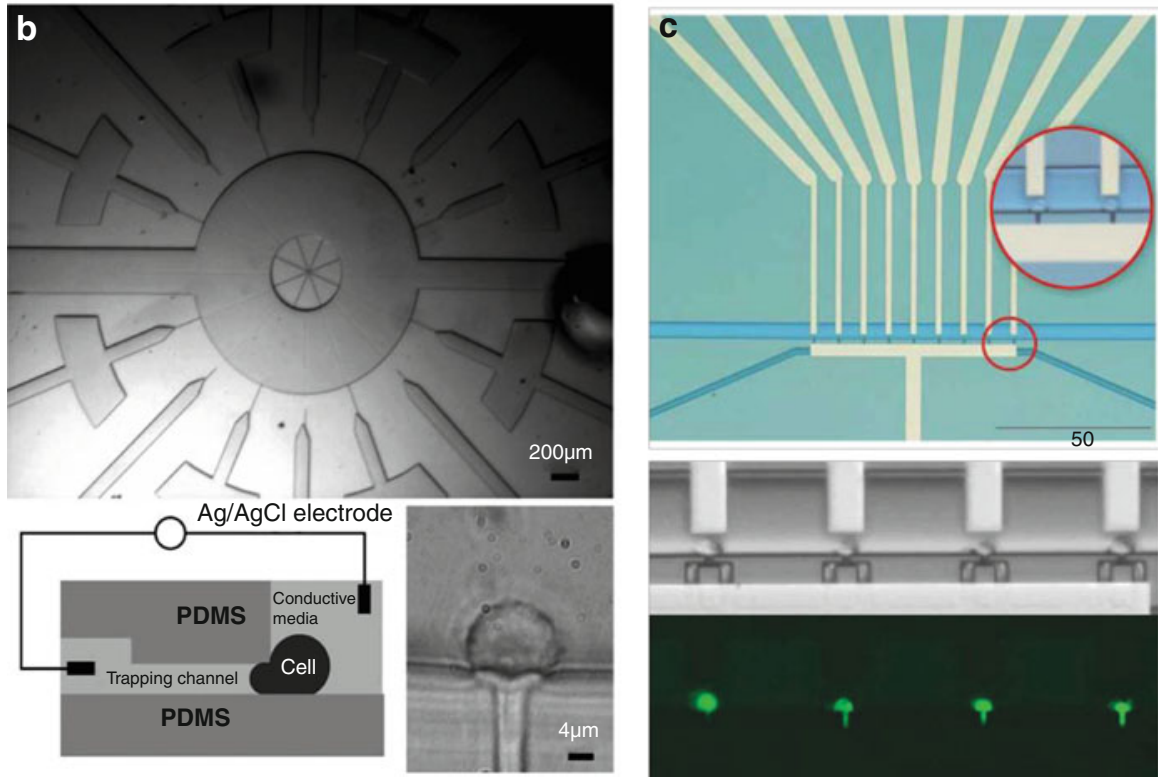

Fig. 2 Cell trapping devices. (a) Three-layer single-cell electroporation device consisting of two microfluidic chambers in silicon, separated by a $1-\mu \mathrm{m}$-thick silicon nitride membrane containing an aperture (2-10 $\mu \mathrm{m}$ in diameter) on which the cell is trapped before electroporation. The top and bottom electrodes are formed by a transparent $\mathrm{n}+$ polysilicon membrane (Courtesy Prof. Rubinsky). (b) Top: Top view of a device for lateral cell trapping. Two wide inlets on either side of the large chamber in the middle are employed to fill it with the solution containing the cells. By applying a suction force via the small radiating side channels, cells are trapped at the channel entrances. The electrodes employed for electroporating the cells and detecting the increase in membrane conductance are connected via the large chamber and to the small cell trapping channels, enabling singlecell electroporation. Bottom left: Schematic cross section of the chip displaying a trapped cell and the two electrodes employed to generate the required field. The trapped cell is pulled into the small opening due to the applied suction force. Bottom right: Photograph of a trapped cell (Reproduced from (Khine et al. 2005) with permission of The Royal Society of Chemistry). (c) Top: Top view of a 
foreign substances into the cells, after pore formation, using electrophoresis (Ionescu-Zanetti et al. 2008).

\section{Advantages and Limitations}

The main advantages of these single-cell miniaturized electroporation devices are the use of milder electrical parameters and the higher control on the poration process, which altogether enhances the success yield in cellular treatment. Since the electrodes are very close to each other and the cells can be tightly trapped, the required potentials become very low compared to conventional systems, ranging from $20 \mathrm{~V}$ (Huang and Rubinsky 2001) down to ca. 1 V (Khine et al. 2005), while the success rate can go up to $100 \%$ (Ionescu-Zanetti et al. 2008). Moreover, plasmids can be actively brought into the cells by electrophoresis (Ionescu-Zanetti et al. 2008), which is not possible in bulk electroporation. Furthermore, the electric field can be tuned to match the cell properties, which further increases the poration yield. Finally, the electric field distribution across the trapped cell can easily be modeled; the cells are considered as spherical objects (Khine et al. 2005; Valero et al. 2008) (Fig. 2b, c), and their position in the electric field is well defined. These modeling aspects also ultimately contribute to the improvement of the poration treatment. All these advantages make these (single)-cell trapping devices the best suited for the treatment of rare cells.

An important drawback of these devices is their level of complexity: they include multiple channels or chambers with low micrometer-sized trapping structures and, sometimes, integrated electrodes. Therefore, their realization involves sophisticated fabrication processes. However, the main limitation of (single)-cell electroporation devices is their treatment capacity; they mostly include a small number of traps, and this, in turn, gives a low cell treatment throughput. It is still worth noticing that a few recent devices including $2 \mathrm{D}$ arrays of micro- and nanopores have scaled up the process to tens of thousands of cells in parallel (Chang et al. 2016 ).

\section{Flow-Through Electroporation Devices}

\section{Principle}

A second category of microfluidic devices for cellular electroporation, which addresses this issue of throughput, consists of flow-through systems (Bao et al.

Fig. 2 (continued) silicon-glass microfluidic single-cell electroporation device. Two microfluidic channels are connected by nine cell traps where cells are captured with the help of a suction force. The electroporation signal is individually applied to each cell with nine stimulation electrodes located above the traps and the common ground electrode placed below the traps. The inset shows a picture of trapped single cells between the electrodes. Bottom: Bright-field and fluorescent microscopy images of four trapped cells $(\mathrm{C} 2 \mathrm{C} 12)$ before and $24 \mathrm{~h}$ after transfection with eGFP (enhanced green fluorescent protein), respectively. The transfection resulted in a $100 \%$ success rate (Reproduced from (Valero et al. 2008) with permission of The Royal Society of Chemistry) 
2008; Kim et al. 2007; Wang and Lu 2008). In these devices, a cell suspension is continuously perfused in a microchannel, and the cells are exposed one by one to the electrical treatment, the voltage being applied again using integrated or external electrodes. With this approach, cells are still treated individually, but larger cell populations can be processed compared to the single-cell electroporation devices. Cells can also be collected at the outlet of the device for further and off-line studies. The channels or structures in which cells are flowing comprise most of the time geometric constrictions, in which the electric field is locally enhanced, so that electroporation takes place in those areas of higher electric field. Therefore, the flow rate at which the cell suspension is perfused in the system is essential, since it directly relates to the time the cells are exposed to the electric field and the electroporation treatment strength and thus, in turn, to the success yield and cell viability rate (Kim et al. 2007). Specifically, increasing the flow rate translates into shorter exposure of the cells to the electric field, which is equivalent to the use of shorter pulses.

Flow-through devices can be further divided into two subcategories, depending on how the cell suspension is flown: (i) using a continuous column of liquid or (ii) using a discrete flow based on droplet microfluidics, where (single) cells are encapsulated in sub-nanoliter aqueous droplets in a continuous flow of an immiscible liquid (i.e., an oil phase).

\section{Continuous Flow Approach}

A first option relies on the use of a simple microchannel with electrodes placed at a short distance from each other, to locally create - between the electrodes - a high electric field.

Most of the flow-through devices include in their design constricted areas, where the electric field is concentrated (Bao et al. 2008; Wang and Lu 2006) (Fig. 3a). The electric signal in this configuration is mostly applied using external electrodes, which are inserted in the reservoirs of the device. The width ratio between the channel and the constricted areas determines the electric field enhancement at the poration area (s). The length of the constricted area and the flow rate define the pulse length for the electrical treatment cells are exposed to. Furthermore, multiple pulses can be applied by implementing a series of constrictions in a single channel.

In case electrodes are integrated, a design with both electrodes on the same substrate is preferred from a fabrication point of view, even if it gives an inhomogeneous distribution in the electric field. If electrodes are placed on the bottom and top substrates, a more homogeneous electric field is created, but their fabrication is more delicate, especially for the alignment of the electrodes. Furthermore, in this configuration, electrodes are obstructing the view so that the electroporation process cannot be monitored in real time. Another configuration of integrated electrodes has been proposed to solve both issues, where electrodes were placed at the half height of the channel (Sukas et al. 2014) (Fig. 3b). A last alternative, which yields better results in terms of uniformity and strength of the electric field while providing optical access, relies on the use of 3D electrode structures. Liquid-based coupling strategies have also been reported to apply a homogeneous electric field through the 

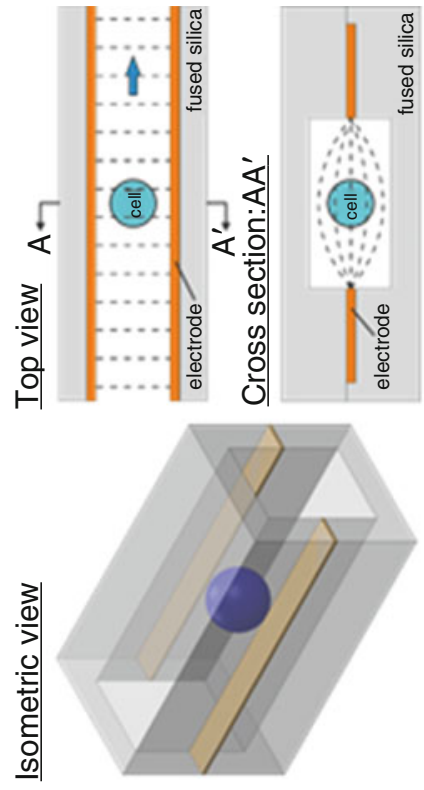

ค

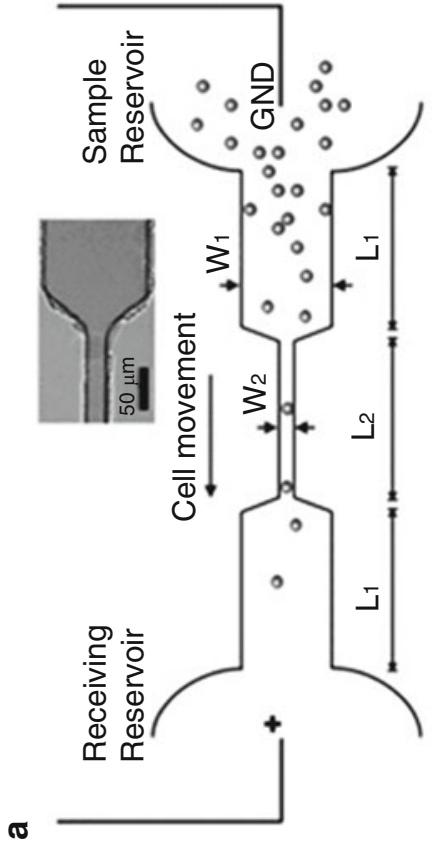

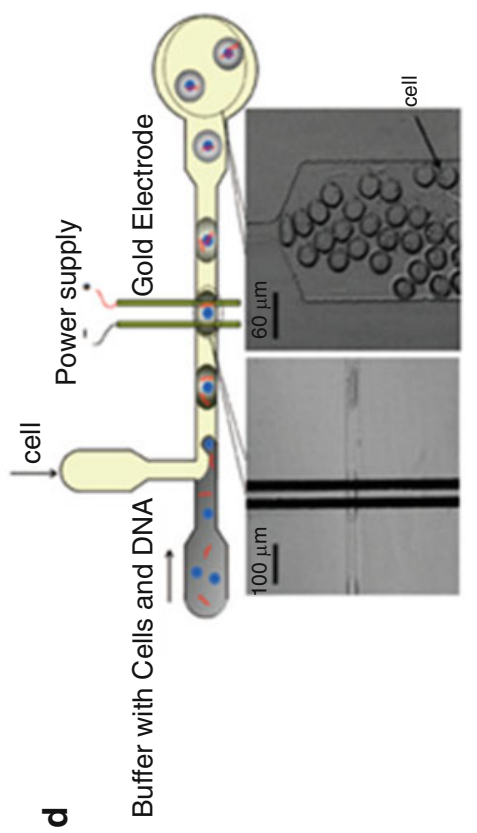

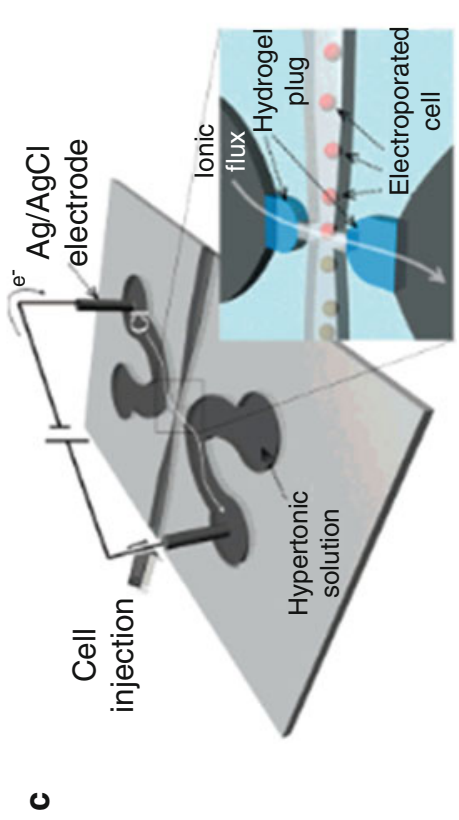

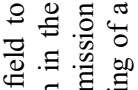

.ํํำ

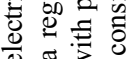

도요 巳ु

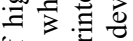

० की

하열

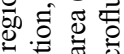

๙ 式

चँ के क्ष

ปั

ㅇㅇㅇ

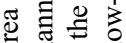

ब

芯导

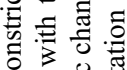

8 .

공흐

船要递

辛.

言卷

D

氜氙导 0.0

苞完

政

홍

馬

$\exists$

눙. 응

. 해 है

氙

롤

设它

응. 용

क्षे है 권

280.00

도용 过 进苛 我氠

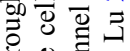
声坖

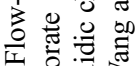
m 흘

$m$ 일

ㅇํㄴ 웡 
cell suspension while using external electrodes and avoiding any direct cellelectrode contact. In those cases, the electric field is enhanced at the intersection between the main channel, in which the cell suspension is perfused, and a side channel in which the electrodes are introduced. In a first liquid-based strategy, the cell suspension is hydrodynamically focused as a single-cell line in the main channel using lateral co-flows of a high ionic strength solution (Zhu et al. 2010); cells are exposed to the electric field when passing the channel intersection, and the voltage drop across the cells is determined by the width of the focused flow. Alternatively, a fluidic junction using a polyelectric salt bridge was created between the main channel where cells flow and the side channels which were filled with a high ionic strength buffer (Kim et al. 2007); here the main channel was made narrower at the channel intersection to further enhance the electric field at the place of cell electroporation (Fig. 3c).

\section{Droplet Microfluidic Approach}

Another class of flow-through microfluidic devices uses a discrete flow, which is also known as droplet microfluidics (Baroud et al. 2010). In droplet microfluidics, an emulsion is created between two immiscible liquids - an aqueous phase and an oil phase - to yield water-in-oil or oil-in-water emulsions, with both an exquisite control on the droplet size and a very high production frequency of more than 1000 droplets/s (Baroud et al. 2010). Thereby, an extremely large number of identical picoliter-tonanoliter-sized droplets reactors are created in a very short period of time. Water-inoil emulsions have found a variety of applications in biology (Baroud et al. 2010). They particularly present a number of advantages for single-cell experimentation such as (i) the ability to confine one cell and its environment in a volume 100-1000 times larger than the volume of a cell and (ii) the massive parallelization of the assays. As such, droplet microfluidics allows high-throughput electroporation of individual cells, which are encapsulated in a small aqueous volume in a continuous nonconductive oil phase (Fig. 3d). In droplet microfluidic devices, electrodes are

Fig. 3 (continued) fused silica microchannel equipped with integrated electrodes placed at half height of the channel to provide an homogeneous electric field across the channel while allowing optical inspection of the cells during their exposure to the electric field (Reproduced from (Sukas et al. 2014) with permission of The Royal Society of Chemistry). (c) Schematic drawing of the electroporation chip including hydrogel plugs that function as salt bridges to form an integrated electrode junction in the channel. Cells are exposed to the electric field and porated in the region between the salt bridges (Reprinted with permission from (Kim et al. 2007). Copyright 2007 American Chemical Society). (d) Droplet microfluidics platform for cell electroporation. Using a T configuration, a water-in-oil emulsion is created with high control on the aqueous droplet size. Each droplet contains one single cell - or no cell - as well as a well-defined amount of DNA to be loaded into the cells. The device includes two electrodes for cell electroporation in the droplets and cell transfection (Reprinted with permission from (Zhan et al. 2009). Copyright 2009 American Chemical Society) 
mostly integrated. As before, the electrical treatment depends on the number of electrode pairs (which correlates with the number of applied electric field pulses on the cells), the distance between the electrodes, the strength of the applied signal and the flow velocity, as well as on the droplet volume (Zhan et al. 2009).

The confinement offered by droplet microfluidics brings specific advantages compared to other flow-through approaches: a high control on the number of cells and substances to be delivered in the cell, which are encapsulated per droplet, which gives additional control on the molecular delivery process; the possibility to incubate the cells and, for instance, DNA to be transfected in the cell prior to electroporation, which can enhance the transfection efficiency (Qu et al. 2012); the possibility to monitor the cell fate and conduct post-electroporation functional assays at the singlecell level in the droplets; and, for molecular analysis, limited dilution of the molecules extracted from the cell, which is essential for the detection of low-concentration species.

\section{Advantages and Limitations}

The main interest of flow-through devices is the throughput in cell treatment, which can reach values of up to $10^{4}-10^{8}$ cells/min. In flow-through devices, cells are treated individually, and the electroporation efficiency rate is as high as for (single)-cell devices. Similarly, voltages as low as a few volts are required, depending on the exact device geometry and dimensions and on how the electric field is applied.

However, parameters of the electrical treatment cannot really be optimized for each individual cell so that the whole population is exposed to the same treatment. Still, this configuration can be further developed to couple cell characterization to cell electroporation, prior or after application of the electrical treatment. For instance, the cell size and morphology can first be evaluated, e.g., using impedance spectroscopy and another pair of integrated electrodes, to tune the electroporation parameters. Most of the flow-through devices do not provide any active control on molecular delivery, and molecular exchange after cell poration only relies on diffusion phenomena. Another limitation associated with these devices is the difficulty to precisely model the electric field distribution across the cells, as the latter keep a certain degree of freedom in their position and orientation with respect to the electrodes, and most of the time the electric field is not homogeneous through the entire channel cross section. Lastly, this flow-through approach is still not suitable for the treatment of both adherent and rare cells, but it is more appropriate for largescale cellular poration.

\section{Promising Applications of Microfluidics for Cell Electroporation}

Implementing the process of electroporation in microfluidic or miniaturized devices is particularly attractive for certain fields of application, as discussed in the following. Furthermore, depending on the application and the required throughput, one specific category of devices, single cell or flow through, may be preferred. 


\section{Fundamental Research}

Since microfluidic devices bring excellent control on the poration parameters and allow varying them in a precise way, they are ideally suited to conduct fundamental studies on the process of electroporation. Furthermore, cells can be monitored in situ to evaluate the effect of the electrical treatment, both in real time or in a longer term, using either electrical or optical means. This set of assets is instrumental, for instance, in gaining new insight into the processes of pore formation and membrane recovery. Similarly, parameters for the electroporation-based treatment can be screened in one device on multiple cells exposed to different conditions and subsequently optimized for various cell types and applications. For such screening, geometrical variations can be implemented in simple channels (e.g., a tapered shape, different constriction dimensions) to locally or gradually alter the electrical treatment and to ultimately study in situ the influence of specific parameters on the poration outcome.

The combination of cell electroporation and microfluidics can also benefit fundamental research in the field of cell biology, for instance, to elucidate targeted signaling pathways, and the activation of specific signaling pathways has notably been studied using reversible electroporation. For instance, the translocation of specific kinases in a cell has been followed using "electroporative flow cytometry," which involves reversible cell poration and analysis of molecular species that diffuse out of the cells through the created pores (Wang et al. 2008). In another approach, cells were transfected with plasmids coding for a protein of interest extracellular signal-regulated kinase 1 (ERK1) coupled to enhanced green fluorescent protein (eGFP), and the accumulation of the resulting fusion protein in the nucleus was tracked in situ in the device using time-lapse imaging after stimulation of the cells with specific growth factors (Valero et al. 2008). The possibility to porate and transfect cells locally in multicellular constructs such as an embryo is of particular interest in the field of developmental biology to unravel processes involved in the development of embryos and to follow cell migration (Mazari et al. 2014).

\section{Intracellular Delivery}

The combination of microfluidics and electroporation has been explored for loading cells with very different substances, ranging from small molecules such as drugs and siRNA to larger substrates such as DNA, proteins, or even nanoparticles and quantum dots (Sun et al. 2014). A clear benefit brought by miniaturized strategies for the introduction of foreign substances in cells is the possibility to better control the delivery process and to dose the amount of foreign material loaded in the cells. Enhanced DNA delivery can be achieved, for instance, using electrophoresisassisted molecular loading after the cell membrane electroporation (Ionescu-Zanetti et al. 2008). Specifically, a single cell can be trapped in a micro- or even nanometer- 
sized constriction connected to a microchannel, in which the substances to be loaded into the cell are introduced. Upon application of a second low-voltage electrical signal across this channel and constriction and after poration of the cell membrane, the substances are pushed into the cell in a highly controlled manner. Furthermore, using electrophoresis-assisted delivery, the transfected DNA is in a free state in the cytoplasm, so that it is more available for its translocation to the nucleus. Similarly, preincubation of individual cells with DNA in confined volumes created using droplet microfluidics proved not only to enhance the transfer efficiency but also to yield more homogeneous transfection in a cell population (Qu et al. 2012). Furthermore, the need in DNA or other substances to load in the cell is drastically reduced since the volumes involved in microfluidic devices are more than three orders of magnitude smaller than in the traditional electroporation cuvettes. For such cellular delivery applications, both types of devices, single-cell electroporation and flowthrough devices, can be used depending on the throughput required and number of cells to be treated.

The possibility to use milder conditions for cell electroporation, together with the higher success rate, and the ability to manipulate small populations of cells or even single cells make a microfluidic format particularly attractive for the treatment of rare and fragile cells such as stem cells, primary cells, or induced pluripotent stem cells, with plethora of applications in various fields such as regenerative medicine ( $\triangleright$ Chap. 82, "Tissue Engineering with Electroporation") and gene therapy ( $\triangleright$ Chap. 81, "Principles of Electroporation for Gene Therapy"). Similarly, the excellent control on the electroporation parameters brought by the micrometer scale has proven to benefit the electroporation of hard-to-transfect cells (Qu et al. 2012).

\section{Food and Biotechnology Applications}

Flow-through microfluidic devices find multiple applications in the food industry for pulsed electric field (PEF) treatment for the pasteurization of liquid food samples ( $\triangleright$ Chap. 133, "Pulsed Electric Field Treatment for Beverage Production and Preservation") like dairy products and fruit juices. There, all bacteria and microorganisms must be inactivated in the sample, which is achieved through irreversible electroporation. PEF also consists of an attractive low energy-consuming treatment for the permeabilization of microalgae for the extraction ( $\triangleright$ Chap. 37, "Selective Extraction of Molecules from Biomaterials by Pulsed Electric Field Treatment") of various cellular components such as the lipid biomass. As microorganisms and algae are much smaller than mammalian cells, higher voltages must be applied than for other applications, which are accompanied by a significant production of heat through Joule effects. In this context, the advantage of using microfluidic devices, which are characterized by higher surface-to-volume ratios, is linked to their ability to dissipate heat in a more efficient way. A shortcoming however of using microscale systems is their relatively low throughput since these applications require processing of large volumes. 


\section{Single-Cell Analysis}

Microfluidic technology shows great promises for the molecular analysis of single cells due to its ability to accurately manipulate individual cells and small-sized samples down to the low picoliter range (Le Gac and van den Berg 2010). To access a cell molecular content, the plasma membrane must be ruptured. To that end, electroporation, whether it is reversible or irreversible, offers specific advantages compared to conventional chemical approaches: it suppresses issues associated with sample dilution and contamination, which can hamper molecular analysis; it allows on-demand and targeted lysis of certain cells if electrodes are individually addressed; it is ideally suited to look at transient species, since electric pulses can rupture the plasma membrane within milliseconds against seconds to minutes for chemical processes; and the membrane poration process can be tuned to be either reversible and noninvasive or irreversible. When electroporation is combined with microfluidics, the retrieved cellular content can be confined in small volumes for further processing or analysis, in situ or off-line, without any extensive dilution and sample loss.

For instance, capillary electrophoresis has been employed, in a proof-of-concept experiment, to separate two dyes previously loaded into the cells and that were detected by fluorescence (Han et al. 2003). Using droplet microfluidics, the entire content of individual cells can be retrieved in individual micrometer-sized reactors with limited dilution of the cell content. Using this approach, de Lange et al. successfully performed enzymatic assays on the content of individual $E$. coli cells to assess the activity of $\beta$-galactosidase at the single-cell level (de Lange et al. 2016). Finally, of particular interest for single-cell analysis is the so-called technique of "electroporative flow cytometry" (Bao et al. 2008), which relies on the selective extraction of molecules located at the periphery of the cytoplasm after reversible cell poration. Using this technique, controlled extraction of small molecules as well as proteins was reported without compromising the viability of the cells (Zhan et al. 2012).

\section{Conclusions}

Cell electroporation can benefit considerably from its implementation in miniaturized and/or microfluidic devices. The whole process is better controlled, down to the single-cell level or even subcellular level, and the success yield is greatly enhanced to virtually reach $100 \%$. Moreover, the risks associated with the treatment are diminished. Indeed, when the dimensions of the devices are reduced to the micrometer range, much lower voltages are required, and signals as low as $<1 \mathrm{~V}$ have led to successful permeabilization of the cell membrane. These low potential values make the devices not only safer to work with but also less energy-consuming while avoiding significant temperature changes induced by Joule heating. Furthermore, miniaturized devices bring enhanced protocols for molecular exchange, i.e., by assisting intracellular delivery using electrophoresis, for instance, and dosing the 
amount of substances loaded in the cell or by confining the extracted cellular content into sub-nanoliter volumes for their analysis or further processing.

Microfluidic electroporation devices can be classified into different categories depending on the way the cells are manipulated: whether they are individually trapped in dedicated microstructures, flown in a channel either as a single-cell file between two electrodes or as individual cells encapsulated in sub-nanoliter droplets. Interestingly, these different types of devices are complementary to each other. Devices from the first category are the best suited for treating single or rare cells and for following the response of each individual cell to the electric field, which is particularly interesting for fundamental studies on the process of electroporation. These devices are also particularly attractive for single-cell study using either imaging of intact and living cells or molecular analysis approaches after cell lysis and extraction of the cellular content. Flow-through devices better apply for middleto-high-throughput treatment of populations of cells. In these devices, cells can be characterized online before and after exposure to the electric field, and they can easily be retrieved from the device for off-line analysis or utilization. Promising applications of flow-through devices are the inactivation of bacteria and microorganisms for the food industry, the transformation of plant cells, or the treatment of algae.

\section{Cross-References}

Assessment of Electroporation by Electrical Impedance Methods

- Critical electric field and transmembrane voltage for lipid pore formation in experiments

- Gene Delivery by Electroporation In Vitro: Mechanisms

- Principles of electroporation for gene therapy

- Pulsed electric field treatment for beverage production and preservation

- Selective Extraction of Molecules from Biomaterials by Pulsed Electric Field Treatment

\section{References}

Bao N, Wang J, Lu C (2008) Microfluidic electroporation for selective release of intracellular molecules at the single-cell level. Electrophoresis 29(14):2939-2944. doi:10.1002/ elps. 200700856

Baroud CN, Gallaire F, Dangla R (2010) Dynamics of microfluidic droplets. Lab Chip 10 (16):2032-2045. doi:10.1039/c001191f

Becker H, Gartner C (2008) Polymer microfabrication technologies for microfluidic systems. Anal Bioanal Chem 390(1):89-111. doi:10.1007/s00216-007-1692-2

Chang LQ, Bertani P, Gallego-Perez D, Yang ZG, Chen F, Chiang CL, Malkoc V, Kuang TR, Gao KL, Lee LJ, Lu W (2016) 3D nanochannel electroporation for high-throughput cell transfection with high uniformity and dosage control. Nanoscale 8(1):243-252. doi:10.1039/c5nr03187g 
Chen JJ, Luo XQ, Wang J, Liao HH, Zhou WL, Zhang L, Han HY, Yu J (2008) Theoretical analysis of T-lymphocytes electroporation model. International Conference on Biomedical Engineering and Informatics. pp 688-690. doi:10.1109/Bmei.2008.149

de Lange N, Tran TM, Abate AR (2016) Electrical lysis of cells for detergent-free droplet assays. Biomicrofluidics 10(2):024114. doi:10.1063/1.4944742

Han FT, Wang Y, Sims CE, Bachman M, Chang RS, Li GP, Allbritton NL (2003) Fast electrical lysis of cells for capillary electrophoresis. Anal Chem 75(15):3688-3696. doi:10.1021/ac0341970

Huang Y, Rubinsky B (1999) Micro-electroporation: improving the efficiency and understanding of electrical permeabilization of cells. Biomed Microdevices 2(2):145-150. doi:10.1023/ A: 1009901821588

Huang Y, Rubinsky B (2001) Microfabricated electroporation chip for single cell membrane permeabilization. Senr Actuators A 89(3):242-249. doi:10.1016/S0924-4247(00)00557-4

Huang Y, Rubinsky B (2003) Flow-through micro-electroporation chip for high efficiency singlecell genetic manipulation. Sens Actuators A 104(3):205-212. doi:10.1016/S0924-4247(03) 00050-5

Ionescu-Zanetti C, Blatz A, Khine M (2008) Electrophoresis-assisted single-cell electroporation for efficient intracellular delivery. Biomed Microdevices 10(1):113-116. doi:10.1007/s10544-0079115-x

Khine M, Lau A, Ionescu-Zanetti C, Seo J, Lee LP (2005) A single cell electroporation chip. Lab Chip 5(1):38-43. doi:10.1039/b408352k

Kim SK, Kim JY, Kim KP, Chung TD (2007) Continuous low-voltage de electroporation on a microfluidic chip with polyelectrolytic salt bridges. Anal Chem 79(20):7761-7766. doi:10.1021/ac071197h

Le Gac S, van den Berg A (2010) Single cells as experimentation units in lab-on-a-chip devices. Trends Biotechnol 28(2):55-62. doi:10.1016/j.tibtech.2009.10.005

Lu H, Schmidt MA, Jensen KF (2005) A microfluidic electroporation device for cell lysis. Lab Chip 5(1):23-29. doi:10.1039/b406205a

Mazari E, Zhao X, Migeotte I, Collignon J, Gosse C, Perea-Gomez A (2014) A microdevice to locally electroporate embryos with high efficiency and reduced cell damage. Development 141 (11):2349-2359. doi:10.1242/dev.106633

Qu B, Eu YJ, Jeong WJ, Kim DP (2012) Droplet electroporation in microfluidics for efficient cell transformation with or without cell wall removal. Lab Chip 12(21):4483-4488. doi:10.1039/ c2lc40360a

Sukas S, Schreuder E, de Wagenaar B, Swennenhuis J, van den Berg A, Terstappen L, Le Gac S (2014) A novel side electrode configuration integrated in fused silica microsystems for synchronous optical and electrical spectroscopy. Lab Chip 14(11):1821-1825. doi:10.1039/ c31c51433a

Sun C, Cao ZN, Wu M, Lu C (2014) Intracellular tracking of single native molecules with electroporation-delivered quantum dots. Anal Chem 86(22):11403-11409. doi:10.1021/ ac503363m

Valero A, Post JN, van Nieuwkasteele JW, ter Braak PM, Kruijer W, van den Berg A (2008) Gene transfer and protein dynamics in stem cells using single cell electroporation in a microfluidic device. Lab Chip 8(1):62-67. doi:10.1039/b713420g

van den Brink FTG, Gool E, Frimat JP, Bomer J, van den Berg A, Le Gac S (2011) Parallel single-cell analysis microfluidic platform. Electrophoresis 32(22):3094-3100. doi:10.1002/elps.201100413

Wang HY, Lu C (2006) Electroporation of mammalian cells in a microfluidic channel with geometric variation. Anal Chem 78(14):5158-5164. doi:10.1021/ac060733n

Wang HY, Lu C (2008) Microfluidic electroporation for delivery of small molecules and genes into cells using a common DC power supply. Biotechnol Bioeng 100(3):579-586. doi:10.1002/ bit. 21784

Wang J, Bao N, Paris LL, Wang HY, Geahlen RL, Lu C (2008) Detection of kinase translocation using microfluidic electroporative flow cytometry. Anal Chem 80(4):1087-1093. doi:10.1021/ ac702065e 
Whitesides GM (2006) The origins and the future of microfluidics. Nature 442(7101):368-373. doi:10.1038/nature05058

Zhan YH, Wang J, Bao N, Lu C (2009) Electroporation of cells in microfluidic droplets. Anal Chem 81(5):2027-2031. doi:10.1021/ac9001172

Zhan YH, Sun C, Cao ZN, Bao N, Xing JH, Lu C (2012) Release of intracellular proteins by electroporation with preserved cell viability. Anal Chem 84(19):8102-8105. doi:10.1021/ ac302462s

Zhu T, Luo CX, Huang JY, Xiong CY, Ouyang Q, Fang J (2010) Electroporation based on hydrodynamic focusing of microfluidics with low dc voltage. Biomed Microdevices 12 (1):35-40. doi:10.1007/s10544-009-9355-Z 